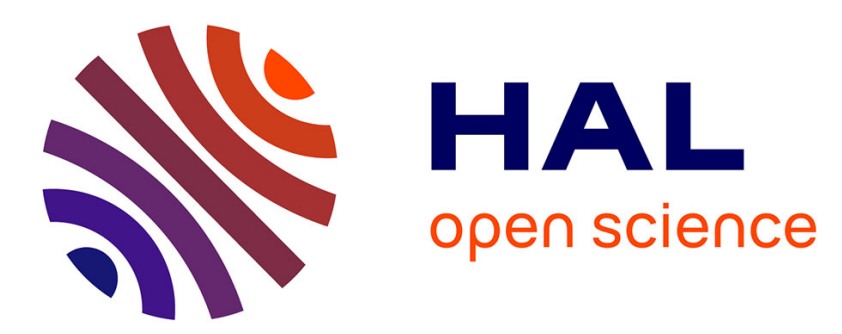

\title{
A Bayesian marked point process for object detection. Application to MUSE hyperspectral data
}

\author{
Aude Costard, Florent Chatelain, Olivier J.J. Michel
}

\section{To cite this version:}

Aude Costard, Florent Chatelain, Olivier J.J. Michel. A Bayesian marked point process for object detection. Application to MUSE hyperspectral data. ICASSP 2011 - IEEE International Conference on Acoustics, Speech and Signal Processing, May 2011, Prague, Czech Republic. pp.SPTM-L2.6. hal-00642152

\section{HAL Id: hal-00642152 \\ https://hal.science/hal-00642152}

Submitted on 17 Nov 2011

HAL is a multi-disciplinary open access archive for the deposit and dissemination of scientific research documents, whether they are published or not. The documents may come from teaching and research institutions in France or abroad, or from public or private research centers.
L'archive ouverte pluridisciplinaire HAL, est destinée au dépôt et à la diffusion de documents scientifiques de niveau recherche, publiés ou non, émanant des établissements d'enseignement et de recherche français ou étrangers, des laboratoires publics ou privés. 


\title{
A BAYESIAN MARKED POINT PROCESS FOR OBJECT DETECTION. APPLICATION TO MUSE HYPERSPECTRAL DATA
}

\author{
F. Chatelain, A. Costard, O. J. J. Michel \\ Gipsa-lab, Images and Signal Department \\ University of Grenoble and Grenoble Institute of Technology, \\ 961 rue de la Houille Blanche, BP 46, 38402 Saint Martin d'Hères, France
}

\begin{abstract}
Marked point processes have received a great attention in the recent years, for their ability to extract objects in large data sets as those obtained in e.g. biological studies or hyperspectral remote sensing frameworks. This paper focuses on an original Bayesian point process estimation for the detection of galaxies from the hyperspectral MUSE data 'cube'. It is shown that this approach allows to obtain a a synthetic representation of the detection problem and circumvent the computational complexity inherent to high dimensional pixel based approaches. The reversible jump Monte Carlo Markov Chain (RJMCMC) implemented to sample the parameters is detailed, and the results obtained on benchmark data mimicking the real instrument are provided.
\end{abstract}

Index Terms - Hierarchical Bayesian Models, Point Processes, Hyperspectral Data, Galaxy Detection.

\section{INTRODUCTION, DATA DESCRIPTION}

The overwhelming growth in size and complexity of experimental data from research fields such as e.g. biology, remote sensing, astrophysics requires to develop new approaches to warrant optimal scientific exploitation of the instrument. This paper focuses on the automated object detection task, formulated in a Bayesian marked point process detection framework.

A marked point process is a stochastic process whose realizations are random configurations of objects. Thus an object is modeled as a point, associated with some marks that accounts for geometrical or spectral features. These processes yield a natural sparse representation for a configuration of a random number of objects in an image. The number of parameters actually depends on the effective number of objects in the current configuration, allowing one to obtain a synthetic representation of the problem. It appears to be a useful tool to circumvent the computational complexity due to high dimensional space problems inherent to pixel-wise approaches. These processes have received a great attention during the last several years for object extraction from biological ([1]) or remotely sensed images (see e.g. [2], [3]) A known drawback of previously developed approaches resides in their high sensitivity to some hyper-parameters that controls the prior and data energy of the process. Consequently, these latter parameters are customary tuned by hand, to some data specific value. The Bayesian approach proposed in this paper overcomes this difficulty by introducing non-informative priors, possibly with

The first author would like to thank Xavier Descombes and Josiane Zerubia for fruitful discussion about point processes during his post-doctoral fellowship at INRIA Sophia Antipolis. This work was supported by ANR DAHLIA, and P.P.F. ISSO, University of Nice Sophia Antipolis, France. a second level of hierarchy within the Bayesian model. It results in a so-called hierarchical Bayesian model. Finally, the posterior distribution is sampled thanks to a Reversible Jump Markov chain Monte Carlo (RJMCMC) method. This hierarchical Bayesian model leads to a general and robust algorithm where the hyperparameters are estimated thanks to a full data-driven criterion [4]. This is, to our knowledge, the first time that a fully Bayesian marked point process is developed for object recognition. Moreover, for the purpose of galaxy detection in the MUSE data cube, the proposed model is designed to handle the large number of monochromatic images (data cube slices).

MUSE (for Multi unit Spectroscopic Explorer, see [5]) project aims at providing the astronomers with a new generation of optical instrument, capable of simultaneously imaging the sky (in 2D) and measuring the optical spectra of the light received at a given position on the sky. MUSE is planed to be installed on the VLT and to be operational in 2012, and its performances are expected to allow observation of far galaxies up to 100 times fainter than those presently detectable. MUSE will deliver 3D 'data-cube' made of a stack of images recorded at 3578 different wavelengths over the range 465$930 \mathrm{~nm}$. Each 'monochromatic' image represents a field of view of $60 \times 60$ arcsec, recorded with a spatial sampling of 0.2 arcsec 0.2 arcsec. Each record results in a data cube of size $1570 \mathrm{MB}$ encoding 3578 images of $300 \times 300$ pixels, possibly containing thousands of objects (galaxies) existing over different subsets of wavelengths! In the literature [6,7], the galaxy morphology is classically assumed to result from elliptical components. Following this assumption, in this paper, a galaxy is approximated by an elliptic object. The marks correspond to the ellipse parameters and to the intensity vector of the galaxy. Galaxy detection is then performed jointly on the different slices of the hyperspectral cube. Throughout this study, we analyze simulation data mimicking the expected hyperspectral record (in terms of SNR, density of objects, spectral content,...), as provided by CRAL [8] within the ANR Dahlia [9] collaborative project.

This paper is organized as follows. The Bayesian model is described in section 2. The RJMCMC sampler is derived in section 3. Simulations conducted on the MUSE cube are reported in section 4. Conclusions are drawn in section 5.

\section{BAYESIAN MODEL}

Let $\boldsymbol{y}$ be a $M \times 1$ vector corresponding to a vectorized image (at a given wavelength $\lambda$ ) counting $M$ pixels, while $\boldsymbol{u}$ denotes a configuration of objects. Finally, $n(\boldsymbol{u})$ denotes the number of objects in the configuration $\boldsymbol{u}$. 


\subsection{Monochromatic Observation model}

$$
\boldsymbol{y}=\boldsymbol{s}+\mathbf{1} m+\boldsymbol{n}
$$

where $m>0$ is the mean of the astrophysical background intensity, $\mathbf{1}=(1 \ldots 1)^{T}$ is the $M \times 1$ matrix with unit elements, $\boldsymbol{n}$ is a $M \times 1$ centered white noise vector with component variance $\sigma^{2}$, and $s$ is a $M \times 1$ vector that models the device response to a galaxy configuration $\boldsymbol{u}$ in the observed scene. The signal $s$ is expressed as:

$$
\boldsymbol{s}=\sum_{i=1}^{n(\boldsymbol{u})} w_{i} \boldsymbol{x}_{i}
$$

where $\boldsymbol{x}_{i}$ stands for the the response of imaging device to an ellipse $u_{i}$ with unitary intensity; $w_{i}$ is the intensity associated with $u_{i}$. An equivalent matrix formulation yields:

$$
s=\boldsymbol{X} w,
$$

where $\boldsymbol{X}=\left[\boldsymbol{x}_{1} \ldots \boldsymbol{x}_{n(\boldsymbol{u})}\right] \in \mathbb{R}^{M \times n(\boldsymbol{u})}$ and $\boldsymbol{w}=\left(w_{1}, \ldots, w_{n(\boldsymbol{u})}\right)^{T} \in \mathbb{R}^{n(\boldsymbol{u})}$.

\subsection{Hyperspectral Likelihood}

From the observation and noise models above, under the assumption that different spectral bands or slices yield independent observations, the hyperspectral likelihood is obtained as the product of the monochromatic (at $\lambda$ ) likelihoods

$$
f\left(\boldsymbol{y}_{\lambda} \mid \boldsymbol{u}, m_{\lambda}, \sigma_{\lambda}^{2}\right)=\left(\frac{1}{2 \pi \sigma_{\lambda}^{2}}\right)^{\frac{M}{2}} \exp \left(-\frac{\boldsymbol{Z}_{\lambda}^{T} \boldsymbol{Z}_{\lambda}}{2 \sigma_{\lambda}^{2}}\right)
$$

where $\boldsymbol{Z}_{\lambda}=\boldsymbol{y}_{\lambda}-\mathbf{1} m_{\lambda}-\boldsymbol{X}_{\lambda} \boldsymbol{w}_{\lambda}$, and all parameters but $\boldsymbol{u}$ depend on $\lambda$. This stresses on the fact that a configuration is shared by all slices (wavelengths) in a given data cube. In the remainder of the paper, $\lambda$ will be omitted for sake of simplicity. Although the independence of the observations from different slices may seem oversimplified, this holds for sufficiently separated (in the wavelength domain) slices. Possible spectral correlations will not be tackled in this paper.

\subsection{Parameter and Hyperparameter Priors}

A noninformative improper prior is chosen for the pair of parameters $\left(m, \sigma^{2}\right)$ :

$$
p\left(m, \sigma^{2}\right)=\frac{1}{\sigma^{2}} \mathbb{1}_{(0,+\infty)^{2}}\left(m, \sigma^{2}\right) .
$$

A Gaussian prior is introduced for the intensity vector:

$$
\boldsymbol{w} \mid \boldsymbol{u}, \sigma^{2} \sim \mathcal{N}\left(\mathbf{0}, \eta^{2} \sigma^{2}\left(\boldsymbol{X}^{T} \boldsymbol{X}\right)^{-1}\right)
$$

Note that all these priors satisfy a principle of invariance. Any affine transformation of the measurements $\boldsymbol{y}$, or of the signal basis $\boldsymbol{X}$, will transform the posterior distribution, and thus our inferences, in the same way. Moreover, the hyperparameter $\eta^{2}$ represents the signalto-noise ratio (SNR) of the observed scene since it is straightforward to show that $\eta^{2}=\frac{E\left[\boldsymbol{s}^{T} s\right]}{E\left[\boldsymbol{n}^{T} \boldsymbol{n}\right]}$.
Finally, a Bayesian hierarchical structure is proposed, by introducing a hyperprior distribution for the hyperparameter $\eta^{2}$. This hyperparameter is modeled as a conjugate inverse-Gamma distribution $\mathcal{I} \mathcal{G}\left(\alpha_{0}, \alpha_{1} / 2\right)$ :

$$
p\left(\eta^{2}\right) \propto\left(\frac{1}{\eta^{2}}\right)^{\alpha_{0}+1} e^{-\left(\frac{\alpha_{1}}{2 \eta^{2}}\right)} .
$$

The parameters $\alpha_{0}$ and $\alpha_{1}$ are chosen sufficiently small to obtain a vague prior (basically, $\alpha_{0}=\alpha_{1}=10^{-3}$ ).

\subsection{Posterior distribution conditionally on a galaxy configura- tion $u$}

The resulting posterior distribution, conditionally on a configuration $\boldsymbol{u}$, is obtained as:

$$
\begin{aligned}
& p\left(\boldsymbol{w}, m, \sigma^{2}, \eta^{2} \mid \boldsymbol{u}, \boldsymbol{y}\right) \propto\left(\frac{1}{2 \pi \sigma^{2}}\right)^{\frac{M+n(\boldsymbol{u})}{2}+1} \frac{1}{\eta^{2}} \frac{n(\boldsymbol{u})}{2} \\
& \quad \times \exp \left(-\frac{\boldsymbol{Z}^{T} \boldsymbol{Z}+\frac{1}{\eta^{2}} \boldsymbol{w}^{T} \boldsymbol{X}^{T} \boldsymbol{X} \boldsymbol{w}}{2 \sigma^{2}}\right) \\
& \quad \times \frac{\exp \left(-\frac{\alpha_{1}}{2 \eta^{2}}\right)}{\left(\eta^{2}\right)^{\alpha_{0}+1}} \mathbb{1}_{(0,+\infty)^{2}}\left(m, \sigma^{2}\right)
\end{aligned}
$$

Based on (2), one can see that the conditional posterior distribution of the intensity vector $\boldsymbol{w}$ given $m, \sigma^{2}, \eta^{2}, \boldsymbol{u}, \boldsymbol{y}$ is Gaussian with mean and covariance matrix respectively:

$$
\begin{aligned}
& \boldsymbol{\mu}=\left(1+\frac{1}{\eta^{2}}\right)^{-1}\left(\boldsymbol{X}^{T} \boldsymbol{X}\right)^{-1}\left[\boldsymbol{X}^{T}(\boldsymbol{y}-\mathbf{1} m)\right] \\
& \boldsymbol{\Sigma}=\sigma^{2} \frac{\eta^{2}}{1+\eta^{2}}\left(\boldsymbol{X}^{T} \boldsymbol{X}\right)^{-1}
\end{aligned}
$$

It allows one to integrate out the parameter vector $\boldsymbol{w}$ to obtain the following marginalized posterior distribution:

$$
\begin{aligned}
& p\left(m, \sigma^{2}, \eta^{2} \mid \boldsymbol{y}, \boldsymbol{u}\right) \propto\left(\frac{1}{\sigma^{2}}\right)^{\frac{M}{2}+1}\left(\frac{1}{1+\eta^{2}}\right)^{\frac{n(\boldsymbol{u})}{2}} \\
& \times e^{-\frac{(\boldsymbol{y}-\mathbf{1} m)^{T}\left(I-\left(\frac{\eta^{2}}{1+\eta^{2}}\right) \mathbf{x}\left(\boldsymbol{X}^{T} \boldsymbol{X}\right)^{-1} \boldsymbol{X}^{T}\right)(\boldsymbol{y}-\mathbf{1} m)}{2 \sigma^{2}}} \\
& \times \frac{\exp \left(-\frac{\alpha_{1}}{2 \eta^{2}}\right)}{\left(\eta^{2}\right)^{\alpha_{0}+1}} \mathbb{1}_{(0,+\infty)^{2}}\left(m, \sigma^{2}\right) .
\end{aligned}
$$

\subsection{Priors on the configuration}

The galaxy configuration $\boldsymbol{u}=\left\{u_{1}, u_{2}, \ldots\right\}$ is modeled as a marked point process (see [10] for more details about point processes). Each marked point $u_{i}$ corresponds to an ellipse $u_{i}=\left(p_{i}, a_{i}, b_{i}, \omega_{i}\right)$, where $p_{i}$ is the center, $a_{i}, b_{i}$ denotes respectively the first and second axis values and $\omega_{i}$ is the orientation of the first axis. This process is defined by its density with respect to the measure $\mu(\cdot)$ of a unitary Poisson process defined on $W \times K$, where $W \subset \mathbb{R}^{2}$ is the coordinate space of the observed scene and $K=\left[r_{\min }, r_{\max }\right] \times\left[r_{\min }, r_{\max }\right] \times$ $[0, \pi[$ is the mark space. In order to regularize the solution and to avoid multiple detections, a hard core penalization is introduced to prevent overlapping. Let

$$
r\left(u_{i}, u_{j}\right)=\max \left(\frac{\sum_{s \in u_{i} \cap u_{j}} x_{i, s}}{\sum_{s \in u_{i}} x_{i, s}}, \frac{\sum_{s \in u_{i} \cap u_{j}} x_{i, s}}{\sum_{s \in u_{j}} x_{i, s}}\right) \in[0,1]
$$


be the overlapping ratio between the responses of two objects $u_{i}$ and $u_{j}$, where $x_{i, s}$ stands for the object response $\boldsymbol{x}_{i}$ evaluated at location $s$. It yields the following hard core density

$$
h(\boldsymbol{u})=\left\{\begin{array}{l}
0 \text { if it exists } i \neq j \text { such that } r\left(u_{i}, u_{j}\right)>t \\
1 \text { otherwise }
\end{array}\right.
$$

where the threshold $t$ is set to the deterministic value $t=1 / 2$.

Finally, the number of galaxy $n(\boldsymbol{u})$ is governed a priori by a Poisson distribution with mean $\beta$. A second level of hierarchy is introduced with the choice of a Jeffreys noninformative hyperprior on this hyperparameter: $p(\beta)=1 / \sqrt{\beta}$. The resulting prior on the configuration is obtained as:

$$
p(\boldsymbol{u}, \beta) \propto \beta^{n(\boldsymbol{u})-1 / 2} e^{-\beta} h(\boldsymbol{u}) \mu(\boldsymbol{u}) .
$$

Integrating out $\beta$, it yields the following marginalized prior:

$$
p(\boldsymbol{u}) \propto \Gamma(n(\boldsymbol{u})+1 / 2) h(\boldsymbol{u}) \mu(\boldsymbol{u}),
$$

$\Gamma(a)=\int_{0}^{+\infty} t^{a-1} e^{-t} d t$ being the classical Gamma function.

\subsection{Posterior distribution}

The joint posterior distribution on both the configuration $\boldsymbol{u}$ and the parameter vector $\boldsymbol{\theta}=\left(m, \sigma^{2}, \eta\right)$ expresses now as:

$$
p(\boldsymbol{u}, \boldsymbol{\theta} \mid \boldsymbol{y}) \propto p(\boldsymbol{\theta} \mid \boldsymbol{u}, \boldsymbol{y}) p(\boldsymbol{u})
$$

where $p(\boldsymbol{\theta} \mid \boldsymbol{u}, \boldsymbol{y})$ and $p(\boldsymbol{u})$ are defined respectively in (3) and (4).

\section{RJMCMC SAMPLER}

Several kinds of movements are considered in the RJMCMC dynamics [11]. Simple moves just update some model parameters, or object features (location, marks). More complex moves allow for dimensional changes in the model, such that the birth of the death of an object in the current configuration. The different moves defined below are finally randomly selected in the RJMCMC dynamic.

\subsection{Sampling the parameters}

One can deduce from (3) the conditional posterior distributions for each parameter given the other ones. These posterior distributions can be used as proposals, leading to an acceptance ratio equal to 1 . These moves reduce to classical Gibbs moves. Note that the different moves to update the parameters are performed sequentially in the RJMCMC dynamic.

\subsubsection{Sampling the background parameters $\left(m, \sigma^{2}\right)$}

Let $\nu=M-1$, and

$$
\begin{aligned}
\boldsymbol{W} & =I-\left(\frac{\eta^{2}}{1+\eta^{2}}\right) \boldsymbol{X}\left(\boldsymbol{X}^{T} \boldsymbol{X}\right)^{-1} \boldsymbol{X}^{T} \\
\delta^{2} & =\left(\mathbf{1}^{T} \boldsymbol{W} \mathbf{1}\right)^{-1}, \quad \tilde{m}=\delta^{2} \mathbf{1}^{T} \boldsymbol{W} \boldsymbol{y} \\
s^{2} & =\nu^{-1} \delta^{2}\left[\boldsymbol{y}^{T} \boldsymbol{W} \boldsymbol{y}-\delta^{2}\left(\mathbf{1}^{T} \boldsymbol{W} \boldsymbol{y}\right)^{2}\right] .
\end{aligned}
$$

Based on (3) and (6), straightforward computations lead to the following Inverse-Gamma density for the variance parameter: $\sigma^{2} \mid\left(m, \lambda^{2}, \boldsymbol{y}, \boldsymbol{u}\right) \sim \mathcal{I} \mathcal{G}\left(\frac{M}{2}, \frac{1}{2 \delta^{2}}\left(\nu s^{2}+(m-\tilde{m})^{2}\right)\right)$. By integrating out $\sigma^{2}$, the marginal posterior for $m$ becomes:

$$
p\left(m \mid \lambda^{2}, \boldsymbol{y}, \boldsymbol{u}\right) \propto\left(\nu+\left(\frac{m-\tilde{m}}{s}\right)^{2}\right)^{-\frac{\nu+1}{2}} \mathbb{1}_{(0,+\infty)}(m) .
$$

Thus the marginal posterior for $m$ is a positive truncated Student distribution with degree of freedom $\nu$, location parameter $\tilde{m}$, and scale parameter $s: m \mid\left(\lambda^{2}, \boldsymbol{y}, \boldsymbol{u}\right) \sim \mathcal{T}^{+}(\nu, \tilde{m}, s)$.

Finally, sampling from the bivariate posterior for $\left(m, \sigma^{2}\right)$, given $\lambda^{2}, \boldsymbol{y}$ and $\boldsymbol{u}$, is performed in two-steps:

1. draw $m \mid\left(\lambda^{2}, \boldsymbol{y}, \boldsymbol{u}\right) \sim \mathcal{T}^{+}(\nu, \tilde{m}, s)$,

2. draw $\sigma^{2} \mid\left(m, \lambda^{2}, \boldsymbol{y}, \boldsymbol{u}\right) \sim \mathcal{I} \mathcal{G}\left(\frac{M}{2}, \frac{1}{2 \delta^{2}}\left[\nu s^{2}+(m-\tilde{m})^{2}\right]\right)$.

\subsubsection{Sampling the RSB parameter $\eta^{2}$}

From (3), the posterior of $\eta^{2}$ is

$$
p\left(\eta^{2} \mid m, \sigma^{2}, \boldsymbol{y}, \boldsymbol{u}\right) \propto\left(\frac{1}{1+\eta^{2}}\right)^{\frac{n(\boldsymbol{u})}{2}} e^{\left[-\left(\frac{1}{1+\eta^{2}}\right) \frac{\beta}{2 \sigma^{2}}\right]} \frac{e^{-\frac{\alpha_{1}}{2 \eta^{2}}}}{\left(\eta^{2}\right)^{\alpha_{0}+1}},
$$

where $\beta=(\boldsymbol{y}-\mathbf{1} m)^{T} \boldsymbol{X}\left(\boldsymbol{X}^{T} \boldsymbol{X}\right) \boldsymbol{X}^{T}(\boldsymbol{y}-\mathbf{1} m)$. This distribution is not standard and it is not possible to sample according to this distribution directly. However, the generation of $\eta^{2} \mid\left(m, \sigma^{2}, \boldsymbol{y}, \boldsymbol{u}\right)$ can be achieved thanks to a standard Metropolis-Hasting step, by introducing an adequate proposal distribution. It leads to a "so-called" Metropolis-within-Gibbs sampler.

\subsection{Birth and Death moves}

For a given configuration $\boldsymbol{u}, p_{B}(\boldsymbol{u})$ denotes the probability to select the birth move, while $p_{D}(\boldsymbol{u})=1-p_{B}(\boldsymbol{u})$ is the probability to choose the reversible move, i.e. the death move (basically, $p_{B}(\boldsymbol{u})=$ $p_{D}(\boldsymbol{u})=1 / 2$ ).

For a birth move, a new object $v$ is drawn independently from the configuration $\boldsymbol{u}$, according to the reference Poisson process. In the death case, the object $u_{i} \in \boldsymbol{u}$ that is proposed to be removed is uniformly selected, thus with probability $p_{S}\left(u_{i} \mid \boldsymbol{u}\right)=1 / n(\boldsymbol{u})$.

Finally, in the birth case, the proposed configuration is $\boldsymbol{v}=\boldsymbol{u} \cup$ $\{v\}$ and the following Metropolis-Hastings-Green ratio is obtained:

$$
r(\boldsymbol{u}, \boldsymbol{v})=\frac{p_{D}(\boldsymbol{v})}{p_{B}(\boldsymbol{u})} \frac{p(\boldsymbol{v}, \boldsymbol{\theta} \mid \boldsymbol{y})}{p(\boldsymbol{u}, \boldsymbol{\theta} \mid \boldsymbol{y})} p_{S}(v \mid \boldsymbol{v}) .
$$

In the death case, the proposed configuration is $\boldsymbol{v}=\boldsymbol{u} \backslash\left\{u_{i}\right\}$ and the Metropolis-Hastings-Green ratio is:

$$
r(\boldsymbol{u}, \boldsymbol{v})=\frac{p_{B}(\boldsymbol{v})}{p_{D}(\boldsymbol{u})} \frac{p(\boldsymbol{v}, \boldsymbol{\theta} \mid \boldsymbol{y})}{p(\boldsymbol{u}, \boldsymbol{\theta} \mid \boldsymbol{y})} \frac{1}{p_{S}\left(u_{i} \mid \boldsymbol{u}\right)} .
$$

Finally, each one of this moves is accepted with probability $\alpha=$ $\min (1, r(\boldsymbol{u}, \boldsymbol{v}))$.

\subsection{Updating an object of the configuration}

This move can be viewed as the composition of a death and a birth move. In fact, an object $u_{i} \in \boldsymbol{u}$ is uniformly selected with probability $p_{S}\left(u_{i} \mid \boldsymbol{u}\right)=1 / n(\boldsymbol{u})$. A new object $v$ is drawn according to the reference Poisson process. We propose to replace the object $u_{i}$ by the new one $v$ leading to the new configuration $\boldsymbol{v}=\boldsymbol{u} \backslash\left\{u_{i}\right\} \cup\{v\}$. The following Metropolis-Hasting ratio is obtained:

$$
r(\boldsymbol{u}, \boldsymbol{v})=\frac{p_{S}(v \mid \boldsymbol{v})}{p_{S}\left(u_{i} \mid \boldsymbol{u}\right)} \frac{p(\boldsymbol{v}, \boldsymbol{\theta} \mid \boldsymbol{y})}{p(\boldsymbol{u}, \boldsymbol{\theta} \mid \boldsymbol{y})},
$$

and the acceptance rate is $\alpha=\min (1, r(\boldsymbol{u}, \boldsymbol{v}))$. 


\section{RESULTS}

The algorithm was applied to a partially integrated (over $\lambda$ ) the MUSE data cube: instead of considering all 3578 wavelengths, 'thick' slices integrating 8 consecutive wavelengths are considered. This lead to a new data set of size $300 \times 300 \times 448$, where images at some average $\lambda$ are assumed independent. The intensity shape $\boldsymbol{x}_{i}$ associated with the ellipsis $u_{i}$ is modeled as a bivariate Gaussian density $f_{2 D}$ for which $u_{i}$ is a $\alpha$ iso-probability contour defined by $\int_{(x, y) \in u_{i}} f_{2 D}(x, y) d x d y=1-\alpha$.

Probabilities to select the parameter update $P_{\text {Parameter }}$ (see section 3.1 ), the birth/death $P_{\mathrm{B} / \mathrm{D}}$ (see 3.2 ), the object update move $P_{\text {Object }}$ (see 3.3) are set to arbitrary values. Note that a fusion/split move (not discussed in this paper) was added for the computation whose results are shown below. The number of MCMC iterations that has been done is $1.1 \times 10^{7}$. It takes about 250 minutes on a $2.40 \mathrm{GHz}$ (single core) CPU.

Figure 1 shows the obtained parameters vs the wavelengths. These parameters are estimated by taking the MMSE estimate over the last 50000 samples provided by the MCMC. The problem which appears around $\lambda=850 \mathrm{~nm}$ was already noticed by the astronomers who prepared the data. It is noteworthy that the shape of the variance receives some physical interpretation, as it matches the expected behavior of the spectrum when water is present in the atmosphere. This was not given as a prior.

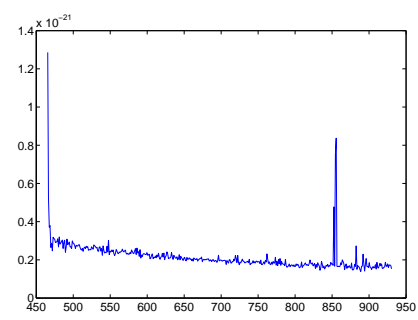

(a)

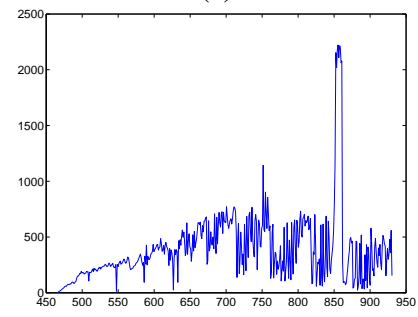

(c)

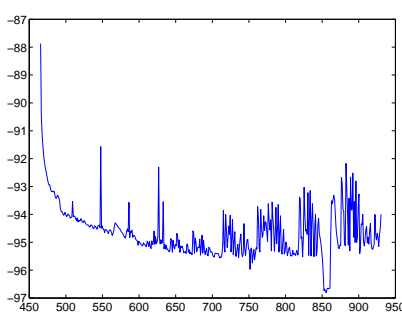

(b)

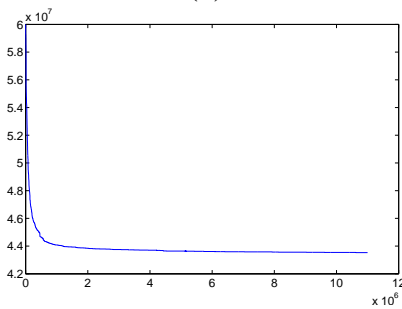

(d)
Fig. 1. Estimated background mean $m$ (a), logarithm of the estimated background variance $\sigma^{2}$ (b), estimated SNR $\eta$ (c) as functions of the central wavelength (in $\mathrm{nm}$ ) for each slice of the data cube. (d): log-posterior density at the current parameter values vs the MCMC iteration.

A projection (integrated over $\lambda$ ) of the detected galaxy configuration is shown on figure 2 .

\section{CONCLUSIONS}

A fully Bayesian marked point process has been studied to automatically extract the galaxies in the MUSE data cube. Future works include the definition of new moves in the RJMCMC dynamic in order to improve the mixing properties of the Markov Chain. The

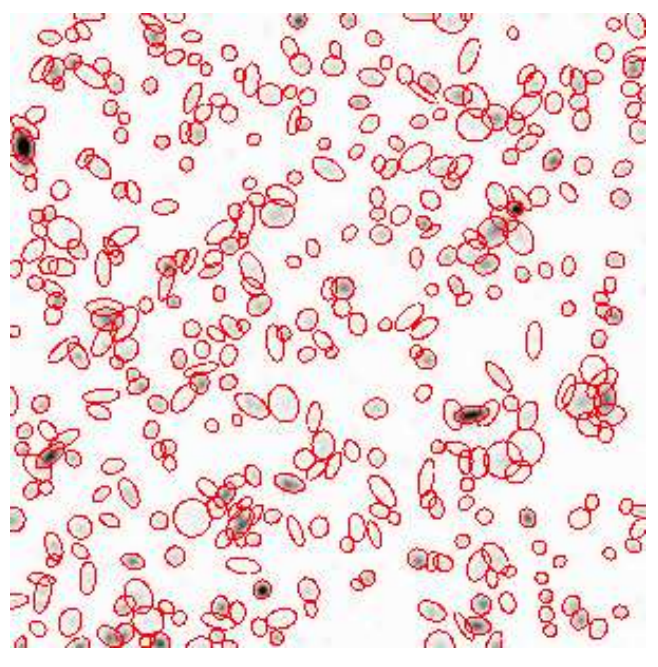

Fig. 2. Projection of the 384 detected galaxies (in negative grayscale colors) and contours (in red)

extension to more complex intensity shape models, taking into account the point spread function of the imaging device must be investigated. Some improvements are also necessary in order to account for very large dynamical variations of the intensity detected for different classes of astrophysical objects.

\section{REFERENCES}

[1] H. Rue and A.R. Syversveen, "Bayesian object recognition with Baddeley's delta loss," Advances Applied Probability, vol. 30, pp. 60-84, 1998.

[2] S. Descamps, X. Descombes, A. Béchet, and J. Zerubia, "Automatic flamingo detection using a multiple birth and death process," in Proc. IEEE International Conference on Acoustics, Speech and Signal Processing (ICASSP'08), 2008, pp. 304-306.

[3] M. Ortner, X. Descombes, and J. Zerubia, "A marked point process of rectangles and segments for automatic analysis of digital elevation models.," IEEE Trans. Pattern Analysis and Machine Intelligence, vol. 30, no. 1, pp. 105-119, Jan. 2008.

[4] Nicolas Dobigeon and Jean-Yves Tourneret, "Bayesian orthogonal component analysis for sparse representation," IEEE Trans. Signal Processing, vol. 58, no. 5, pp. 2675-2685, May 2010.

[5] "Official website of the MUSE project," http://muse.univ-lyon1.fr/.

[6] L. Simard, C. N. A. Willmer, N. P. Vogt, V. L. Sarajedini, A. C. Phillips, B. J. Weiner, D. C. Koo, M. Im, G. D. Illingworth, and S. M. Faber, "The deep groth strip survey. ii. hubble space telescope structural parameters of galaxies in the groth strip," The Astrophysical Journal Supplement Series, vol. 142, no. 1, pp. 1, 2002.

[7] C. Y. Peng, L. C. Ho, C. D. Impey, and H.-W. Rix, "Detailed Structural Decomposition of Galaxy Images," Astron. J., vol. 124, pp. 266-293, 2002.

[8] "Official website of the CRAL laboratory," http://www-obs. univ-lyon 1. fr/? lang=en.

[9] "Official website of the Dahlia project," https://dahlia.oca.eu/ twiki/bin/view/Main/WebHome.

[10] D. Stoyan, W. S. Kendall, and J. Mecke, Stochastic Geometry and Its Applications. Second Edition, John Wiley \& Sons, Chichester, 1995.

[11] Peter J. Green, "Reversible Jump Markov chain Monte Carlo computation and Bayesian model determination," Biometrika, vol. 52, pp. 711-732, 1995. 